\title{
Preattentive guidance of eye movements during triple conjunction search tasks: The effects of feature discriminability and saccadic amplitude
}

\author{
DIANE E. WILLIAMS and EYAL M. REINGOLD \\ University of Toronto, Toronto, Ontario, Canada
}

\begin{abstract}
Eye movements were monitored during the performance of triple conjunction search tasks. Stimuli varied in color, shape, and orientation. Across trials, the target was either present or absent, and displays consisted of 6,12 , or 24 stimuli. Stimulus discriminability was manipulated for the shape dimension, with half of the participants seeing displays of Es and Fs (low-discriminability [LD] condition) and half seeing displays of Cs and Ts (high-discriminability [HD] condition). Participants in both conditions performed two search tasks. In the single-feature (SF) task, the targetstimulus shared one feature with each of the distractors, whereas in the two-feature (TF) task, it shared two features with each distractor. An examination of saccadic endpoints revealed that participants were more likely to fixate on distractor stimuli sharing color (SF task) or color and shape (TF task) with the target. This was a robust finding, being observed across participants, saccades of different amplitudes and sequential position, and following short and long latencies to move. The extent to which participants made use of shape information increased with discriminability.
\end{abstract}

The feature integration theory of attention, as originally formulated (Treisman \& Gelade, 1980; Treisman, Sykes, $\&$ Gelade, 1977), proposed that information from parallel preattentive processes could mediate performance in a visual search task only if the target was defined by the presence of a unique feature (e.g., searching for a red $\mathrm{O}$ among blue and green Os). In contrast, if the target was defined by a specific combination of features, each of which was shared with the distractors (e.g., searching for a red $\mathrm{O}$ among blue Os and red $\mathrm{Xs}$ ), then determining whether the target was present or absent required a serial search of the stimulus display in which the focus of attention was directed at each item in turn. Consequently, in such a search task, although preattentive processes had already determined which features were present in the stimulus display, this information would be inaccessible to the mechanisms directing the attentional focus (see Cave \& Wolfe, 1990; Wolfe, Cave, \& Franzel, 1989). These two types of search task will henceforth be referred to as feature and conjunction search tasks, respectively.

This early version of feature integration theory is inconsistent with the results of several subsequent studies (see Wolfe, 1998, for review). For example, a strictly item-by-item search of the stimulus array is hard to recon-

This research was supported by grants to E.M.R. and to Morris Moscovitch from the Natural Science and Engineering Research Council of Canada. We thank James Hoffman, Peter Braun, Gregory Zelinsky, and an anonymous reviewer for their comments on earlier versions of this manuscript. We also thank Jiye Shen for assistance in data analysis. Correspondence should be addressed to E. M. Reingold, Department of Psychology, University of Toronto, $100 \mathrm{St}$. George Street, Toronto, ON, M5S 3G3, Canada (e-mail: reingold@psych.utorontoca). cile with reports of parallel or highly efficient performance in a variety of conjunction search tasks (e.g., McLeod, Driver, \& Crisp, 1988; Nakayama \& Silverman, 1986; Steinman, 1987; Theeuwes \& Kooi, 1994; Wolfe et al., 1989). This, combined with observations of serial or inefficient performance in feature search tasks (e.g., Nagy \& Sanchez, 1990; Wolfe, Friedman-Hill, Stewart, \& O'Connell, 1992), has led researchers to suggest that both feature and conjunction search tasks vary along a continuum of search efficiency, with the slope of the response time (RT) by display size function increasing as search becomes less efficient (e.g., Wolfe, 1998). Feature integration theory has been amended to address such variations in search efficiency by suggesting that features are coarsely coded, that the attentional focus may vary in size, and that the representations of distractor features not shared with the target can be inhibited (Treisman, 1991, 1993a, 1993b; Treisman \& Gormican, 1988).

Another theory that attempts to account for variations in search efficiency is the guided search theory proposed by Wolfe and his colleagues (e.g., Cave \& Wolfe, 1990; Wolfe, 1994, 1996; Wolfe et al., 1989). According to this theory, during visual search, preattentive processes guide shifts of attention by pinpointing stimulus locations likely to contain the target. This preattentive information encompasses both bottom-up (i.e., how closely a particular item resembles other items in the display) and top-down (i.e., how closely the features of a given item match those characterizing the target) influences. These sources of information combine to form an "activation map," which contains peaks of activity at likely target locations. During the search process, the focus of attention is directed at the stimulus location showing the most activity. If this 
location contains the target, the participant responds that the target is present and search terminates; otherwise, the attentional focus is directed to the next most active location. This process continues until the target is found, until all of the stimuli with activations above a particular threshold have been analyzed, or until search extends beyond a certain time limit; the latter two conditions typically result in negative responses. It should be noted that, according to the guided search theory, the same mechanisms subserve performance in both feature and conjunction search tasks; what differs across tasks is the effectiveness of the preattentive information at guiding the attentional focus to the target location.

If visual search is guided by preattentive information, this guidance may be reflected by patterns of eye movements. Consequently, one of the primary goals of the present experiment was to investigate whether patterns of eye movements show evidence of selectivity during visual search. For example, if search is guided, participants might show a tendency to fixate stimuli sharing a particular feature or features with the target (e.g., color, shape, orientation).

The results from two early studies by L. G. Williams (1967) and by Luria and Strauss (1975) are consistent with this prediction. L. G. Williams created stimuli by combining values from three stimulus dimensions: color, shape (i.e., simple geometric figures), and size. Participants were then asked to search arrays of such stimuli for a particular target stimulus. On some trials, participants were given information concerning one or more of the target features before the stimulus display was presented. Under these conditions, participants showed a strong tendency to direct saccades toward stimuli sharing target color. Information concerning target size had only a slight effect on a participant's choice of saccadic target, whereas the effect of target shape was minimal at best. In the study by Luria and Strauss, participants saw arrays of 16 dials; their task was to look for the only dial that had not been rotated from the starting position. Participants were sometimes informed of the color, shape, or color-shape (CS) combination characterizing the target prior to trial onset. When given such information, participants showed a marked tendency to direct saccades to distractor stimuli sharing target color. A similar, although weaker, effect was observed when participants were told the target shape. Finally, when supplied with information concerning both the color and shape of the target, participants' choice of saccadic targets was guided by both dimensions, although, again, color information had a stronger effect.

More recent studies of eye movements during visual search have yielded conflicting results. In an experiment by Findlay (1997), participants were shown arrays of 16 colored shapes and were instructed to saccade as quickly as possible toward a target stimulus defined by a particular combination of color and shape. In each display, the target shared either shape or color with some distractors, but had neither feature in common with others. On trials where participants mistakenly moved their eyes toward one of the distractor stimuli, it was typically a stimulus that shared one of the target features. These results are consistent with preattentive guidance of the first saccade but reveal nothing about the selectivity of eye movements as search progresses. Additional support for saccadic selectivity throughout the search process was provided by Scialfa and Joffe (1998). They monitored eye movements while participants searched for a target that shared a single feature with each of two distractors (a white bar tilted $45^{\circ}$ to the right among white bars tilted $45^{\circ}$ to the left and black bars tilted $45^{\circ}$ to the right). They found strong evidence of selectivity, with participants being three times more likely to fixate on distractors that shared target contrast than on distractors that did not.

The findings of selectivity reported by Findlay (1997) and by Scialfa and Joffe (1998) conflict with the results of a study by Zelinsky (1996). In this study, participants were asked to search for a green horizontal bar or a red vertical bar among two types of distractor stimuli. "Similar" distractors (green vertical bars and red horizontal bars) each shared one feature with the target, whereas "dissimilar" distractors (yellow bars tilted $45^{\circ}$ to the right and blue bars tilted $45^{\circ}$ to the left) did not. Zelinsky reasoned that if the guidance of search by preattentive processes was reflected by patterns of eye movements, participants should be more likely to direct saccades toward "similar" than toward "dissimilar" distractors. He found only a slight tendency toward such selectivity and concluded that "despite some evidence in support of a process guiding search, the data argue more strongly for a relatively weak influence of selectivity in oculomotor search behavior" (p. 2183). He went on to suggest that other cognitive strategies may play an important role in determining saccadic endpoints. In keeping with this proposal, an analysis of initial saccades revealed that participants showed a marked tendency to direct these saccades to the upper left-hand quadrant of the stimulus array.

Findlay and Gilchrist (1998) have suggested that the weak selectivity observed by Zelinsky (1996) may be due to the structure of the stimulus displays in his experiment. Previous research indicates that selection of the saccadic endpoints during visual search is heavily influenced by the distance between each stimulus in a display and the fovea (e.g., Findlay, 1980). In the experiment by Findlay described earlier, a constant number of stimuli were present in each display, appearing in set eccentricities along the border of one or two imaginary circles. In contrast, the structure of Zelinsky's displays meant that stimuli were presented at a variety of eccentricities. Findlay and Gilchrist have suggested that, as a result, guidance of eye movements in Zelinsky's study may have been more influenced by the proximity of stimuli to the fovea than by the presence or absence of features shared with the target. Zelinsky did not investigate guidance as a function of saccadic amplitude, making it difficult to evaluate this explanation of his results.

Another issue that must be addressed by any attempt to explain inconsistencies across studies is the probability of refixation during visual search. While searching the stimulus array, participants may sometimes refixate 
a particular stimulus by executing a small-amplitude saccade. If such refixations are not separated from other fixations during analysis, they may affect estimates of selectivity. For example, participants might be more likely to refixate distractor stimuli that are more difficult to distinguish from the target. If this is true, it means that they might be more likely to refixate certain classes of distractors as opposed to others. Although this is not an issue in Findlay's (1997) study, where only selectivity of the first saccade was examined, the inclusion of such refixations during the analysis of eye movements may help to account for the differing results reported by Zelinsky (1996) and by Scialfa and Joffe (1998).

One purpose of the present experiment was to determine whether selectivity of eye movements is apparent when saccadic amplitude is taken into account. This would address two of the issues raised above by (1) allowing an evaluation of Findlay and Gilchrist's (1998) hypothesis that proximity to the fovea may override any guidance of visual search by stimulus features, and (2) allowing an analysis of the selectivity of eye movements during visual search that would not be distorted by the presence of refixations. This was done by calculating the length of each saccade in degrees of visual angle and then dividing saccades into one of seven bins depending on size (i.e., $0^{\circ}-1^{\circ}, 1^{\circ}-2^{\circ}, 2^{\circ}-3^{\circ}, 3^{\circ}-4^{\circ}, 4^{\circ}-5^{\circ}, 5^{\circ}-6^{\circ}$, and $>6^{\circ}$ ). The saccades in each bin could then be analyzed separately to look for any evidence of selectivity.

Hooge and Erkelens (1999) have proposed an alternative explanation for the inconsistencies across studies. They monitored eye movements in a visual search task, independently manipulating the difficulty of stimulus discrimination at the fovea and of selecting saccadic targets in the periphery. They found that the likelihood of directing saccades toward a distractor stimulus similar to the target increased with the duration of the preceding fixation. They have suggested that fixation duration is determined by the difficulty of foveal discrimination, with more difficult discriminations leading to longer fixation durations and stronger guidance (the exact level of guidance is then thought to depend on the difficulty of peripheral selection). According to Hooge and Erkelens, the inconsistent findings seen in previous studies of saccadic guidance may be due to differences across tasks in the difficulty of foveal discrimination and of peripheral selection and the associated effects on fixation duration.

Following Hooge and Erkelens (1999), the present study also investigated whether the strength of saccadic selectivity increases as a function of the duration of the preceding fixation. To this end, for each participant, the latencies of all first saccades were divided into those falling below the median (short latencies) and those falling above the median (long latencies). Patterns of guidance could then be compared for saccades following short and long latencies. Although the difficulty of foveal discrimination and the difficulty of peripheral selection were not independently manipulated, as they were in the Hooge and Erkelens study, a straightforward prediction can be made on the basis of their results. If guidance does in- crease with fixation duration, stronger evidence of selectivity should be observed following long initial latencies than following short initial latencies. Initial latencies, as opposed to fixation durations, were examined in this analysis because it was felt that latency to move would be less ambiguous with regard to the onset of stimulus information and to the temporal window available for saccadic programming.

Another goal of the present experiment was to investigate whether search could be simultaneously guided by information from more than a single stimulus dimension, as predicted by the guided search theory (e.g., Wolfe, 1994, 1996, 1998). To this end, we investigated patterns of eye movements during two triple conjunction search tasks. In such visual search tasks, the target and distractor stimuli are created by combining features from three stimulus dimensions, allowing the experimenter to vary the number of features shared by the target and distractor stimuli. In the present experiment, these dimensions were color, shape, and orientation. In the single-feature (SF) task of the present experiment, the target stimulus shared one feature with each of three distractor stimuli (i.e., color, shape, or orientation), whereas in the twofeature (TF) task, the target shared two features with each of three distractors (i.e., color and shape, color and orientation, or shape and orientation). The findings from the SF task could be used, therefore, to derive estimates of the contribution of single stimulus dimensions to guidance. The TF task, on the other hand, could be used to get estimates of the relative contributions of pairs of stimulus dimensions to saccadic selectivity. As a result, a comparison of guidance across these two conditions may help to determine whether performance in the TF task is consistent with simultaneous guidance as opposed to being a simple summation of guidance estimates for single dimensions derived in the SF task.

The final goal of the present study was to examine the effect of stimulus discriminability on guidance of visual search. To this end, in both the SF and TF search tasks we manipulated stimulus discriminability for the shape dimension. In the low-discriminability (LD) search condition, the stimuli were Es or Fs while in the high-discriminability (HD) search condition, the stimuli were Cs or Ts. According to the guided search theory by Wolfe and his colleagues, preattentive information becomes more effective in the guidance of the attentional focus to the target as stimulus discriminability increases (Wolfe, 1994, 1998). Thus, it can be predicted that shape information should have a greater impact on guidance in the HD condition than in the LD condition.

\section{METHOD}

\section{Participants}

Two groups of 32 participants took part in the present experiment (23 males and 41 females). Participants in the first group saw LD displays, while participants in the second group saw HD displays. Participants ranged in age from 19 to 37 years. Participants were tested individually, and all had normal or corrected-to-normal vision. They were paid $\$ 10$ an hour. 


\section{Apparatus}

The eyetracker employed in this research was the SR Research Ltd. EyeLink system. This system has high spatial resolution $\left(0.005^{\circ}\right)$ and a sampling rate of $250 \mathrm{~Hz}$ (4-msec temporal resolution). The EyeLink headband has three cameras, allowing simultaneous tracking of both eyes and of head position for head-motion compensation. By default, only the participant's dominant eye was tracked in our studies. The EyeLink system uses an Ethernet link between the eyetracker and display computers for real-time saccade and gaze position data transfer. In the present study, the configurable acceleration and velocity thresholds were set to detect saccades of $0.5^{\circ}$ or greater.

Stimulus displays were presented on two monitors, one for the participant (a 17-in. Viewsonic 17PS) and one for the experimenter. The experimenter monitor was used to give feedback in real time about the participant's computed gaze position. This feedback was given in the form of a cursor measuring $1^{\circ}$ in diameter that was overlaid on the same image being viewed by the participant. This allowed the experimenter to evaluate system accuracy and to initiate a recalibration if necessary. In general, the average error in the computation of gaze position was less than $0.5^{\circ}$ of visual angle.

\section{Materials and Design}

Stimuli were created by combining features on three dimensions - color (red vs. blue), shape (E vs. F in LD displays and C vs. $\mathrm{T}$ in HD displays), and orientation (upright, or vertical, vs. rotated $90^{\circ}$ clockwise, or horizontal). This made a total of eight different stimuli in the LD and HD conditions (LD condition: red, vertical E; blue, vertical E; red, vertical F; blue, vertical F; red, horizontal E; blue, horizontal E; red, horizontal F; and blue, horizontal F; HD condition: red, vertical C; blue, vertical C: red, vertical T; blue, vertical $\mathrm{T}$; red, horizontal $\mathrm{C}$; blue, horizontal $\mathrm{C}$; red, horizontal $\mathrm{T}$; and blue, horizontal $\mathrm{T}$ ). Individual letters subtended $1.5^{\circ}$ horizontally and vertically. Colors were matched in luminance and saturation $(x, y$ CIE coordinates: $0.601,0.322$ [red] and 0.151, 0.078 [blue] ). Participants in both the LD and HD groups performed two search tasks: an SF task and a TF task. In the SF task, the target shared one feature (color, shape, or orientation) with each of three types of distractors, whereas in the TF task, it shared two features (color-shape [CS], color-orientation [CO], or shape-orientation [SO]) with each of three types of distractors. In both SF and TF search tasks, each distractor stimulus had one feature in common with each of the other distractor stimuli. For any given participant, the identity of the target stimulus remained constant across tasks (SF and TF). Thus, within a given group (LD or HD), each of the eight potential target stimuli was assigned to 4 participants. This meant that each stimulus appeared with equal frequency as the target in both the SF and TF search tasks. Each stimulus also appeared equally frequently as a distractor in the two tasks.

Displays were composed of 6,12 , or 24 stimuli and were created using an imaginary $6 \times 6$ grid of stimulus positions. This grid subtended $12^{\circ}$ both horizontally and vertically and contained 16 possible target locations (the four positions in each corner of the grid). For all three display sizes, positive arrays were created by assigning the target to one of these 16 possible locations; the appropriate number of distractor stimuli $(5,11$, or 23$)$ were then randomly assigned to the other 35 positions. Three different stimulus configurations were created for each target position in each display size. Negative displays were then created by replacing the target in each positive display with a distractor. This method of creating displays generated 48 unique stimulus displays (16 target positions $\times 3$ displays per position) for each trial type (positive vs. negative) by display size $(6,12$, or 24$)$ combination, for a total of 288 displays. Each of the three distractor stimuli appeared an equal number of times across displays for each condition (i.e., positive and negative displays of size 6,12 , or 24). The same stimulus configurations were used as displays in both the SF and TF search tasks in both the LD and HD search conditions, with the particular target and distractor stimuli changing, as appropriate, across both conditions and tasks.

For each participant, the 288 displays were randomly assigned to blocks of 96 trials each, with the restriction that each block contain an equal number of trials at each display size $(6,12$, or 24$)$ and each trial type (positive or negative). The order of the stimulus displays within each block was then randomized under the condition that there be no more than 4 displays of a given size or trial type in a row. Each participant saw a unique order of displays in each search task.

Eighteen practice displays were also created, with 3 displays for each trial type by display size combination; the same stimulus configurations were used in both LD and HD displays for both search tasks (SF or TF). Two random orders of practice trials were generated, one for each task, with the same orders being used in both the LD and HD conditions.

\section{Procedure}

The experiment was run in a lighted room with a luminance of approximately $30 \mathrm{~cd} / \mathrm{m}^{2}$. Each participant performed both SF and TF visual search tasks; the order of these two tasks was counterbalanced across participants within each search condition (LD vs. HD). Before beginning the first search task, participants were informed that stimulus displays would consist of 6,12 , or 24 letters and were told the identity of the target and distractor stimuli. They were told that the target stimulus would not be present on all trials and that their task was to look for the target stimulus in each display, responding as quickly and as accurately as possible. Participants then performed the 18 practice trials for the first type of search task, followed by the three blocks of experimental trials. Following completion of the first task, the instructions for the second visual search task were given. Participants were told that they would see more displays containing 6,12 , or 24 letters. They were informed that they would be looking for the same target stimulus, but that there were three new distractor stimuli. Participants then performed the 18 practice trials and 288 experimental trials in the second search condition.

At the beginning of each trial, a fixation point was presented in the center of the computer screen in order to correct for drift in gaze position (see Stampe, 1993). Two hundred milliseconds following drift correction, a stimulus display would appear in the center of the screen, where it remained visible until the participant responded. Participants held a response pad in their laps with the thumb of their dominant hand resting between two buttons; these two buttons were positioned one above the other. Participants pressed one of these buttons if the target was present, the other if it was absent. The particular button used to respond "yes" on positive trials was counterbalanced across participants. The time between display onset and the participant's response was recorded as the RT.

\section{RESULTS AND DISCUSSION}

Trials were dropped from analysis if a saccade or blink overlapped display onset or if a blink occurred within $300 \mathrm{msec}$ after display onset. These criteria meant that $2.8 \%$ of trials in the SF search task and $3.9 \%$ of trials in the TF search task were excluded from analysis. Trials on which participants responded incorrectly were also excluded from analysis (1.2\% of trials in the SF search task and $4.3 \%$ of trials in the TF search task); there was no evidence of a speed-accuracy tradeoff. Also excluded from analysis were trials on which a participant's response time fell more than $2 S D$ above or below that participant's mean in the appropriate trial type (positive vs. negative) by display size $(6,12$, or 24$)$ condition $(4.7 \%$ of trials in 
the SF search task and 3.8\% of trials in the TF search task). The remaining data were analyzed using analyses of variance (ANOVAs). Main effects and lower-order interactions will only be discussed in the absence of a significant higher-order interaction. Post hoc comparisons were carried out using the Newman-Keuls test with $\alpha=.05 .^{1}$

\section{RT and the Number of Fixations per Trial}

Although the data concerning guidance of eye movements were of primary interest, RT and the number of fixations per trial were also examined to confirm that performance in the present experiment was consistent with the results of previous studies of triple conjunction search tasks. RT and the number of fixations per trial were analyzed using ANOVAs that crossed the between-subjects variable of discriminability (LD vs. HD) with the withinsubjects variables of search type (SF vs. TF), trial type (positive vs. negative), and display size $(6,12$, or 24$)$. The search type $\times$ trial type $\times$ display size interaction was significant for both RT and NF [RT: $F(2,124)=$ 142.96, $M S_{\mathrm{e}}=5,807.4, p<.001$; number of fixations: $\left.F(2,124)=125.92, M S_{\mathrm{e}}=0.12, p<.001\right]$. An examination of Figure 1 reveals that, under both LD and HD conditions, average RT and mean number of fixations per trial increased with display size, with this increase being more pronounced on negative trials than on positive trials and greater for the TF than for the SF search task. As demonstrated in previous studies (Quinlan \& Humphreys, 1987; Treisman \& Sato, 1990; Wolfe et al., 1989), search was more efficient in the SF than in the TF task.

The discriminability $\times$ search type $\times$ trial type $\times$ display size interaction was also reliable for RT and marginally significant for number of fixations [RT: $F(2,124)=$ 6.09, $M S_{\mathrm{e}}=5,807.4, p<.005$; number of fixations: $\left.F(2,124)=2.63, M S_{\mathrm{e}}=0.12, p=.08\right]$. This interaction reflects the fact that, despite the similar results observed in the LD and HD conditions, the increase with display size in RT and number of fixations is more pronounced in the LD condition (Figure 1). This finding is consistent with predictions from the guided search theory (see Wolfe, 1994, 1998) and indicates that our manipulation of discriminability was successful.

\section{Guided Search}

Selectivity of participants' eye movements was determined in a manner similar to that used by Zelinsky (1996). For every trial, the distance in degrees of visual angle was calculated between the fixation following each saccade and every stimulus in the display. The stimulus closest to the fixation was taken to be the target of that saccade. Once a fixation had been assigned to a particular stimulus, that stimulus could be examined to see on what dimension, or pair of dimensions, this stimulus matched the target. For each participant, the number of fixations successfully assigned to distractors was then summed for each dimension in the SF task (color, shape, or orientation) or pair of dimensions in the TF task (CS, CO, or $\mathrm{SO})$. Because a distractor could only match the target on a single dimension in the SF task, whereas it could match the target on a pair of dimensions in the TF task, separate analyses were carried out for the two search tasks.

As pointed out by Zelinsky (1996), the results from negative trials can be interpreted more clearly than those on positive trials, where the presence of the target may influence search behavior. Thus, only negative trials were included in this analysis. Across participants, 12,206 (LD condition) and 9,070 (HD condition) fixations were successfully assigned to distractor stimuli in the SF search task, whereas 30,310 (LD condition) and 18,585 (HD condition) fixations were assigned in the TF search task.

SF search task. The first step in the guided search analysis was to assign each saccade to one of seven bins according to size (i.e., $0^{\circ}-1^{\circ}, 1^{\circ}-2^{\circ}, 2^{\circ}-3^{\circ}, 3^{\circ}-4^{\circ}, 4^{\circ}-5^{\circ}$, $5^{\circ}-6^{\circ}$, and $>6^{\circ}$ ). This was done to determine whether selectivity, if observed, was apparent for saccades of different sizes. For each bin, the proportion of fixations assigned to distractors sharing target color, shape, or orientation was then calculated for each participant. These proportions were analyzed using a series of three ANOVAs. The first ANOVA contrasted the proportion of fixations assigned to target color with that assigned to target shape, the second compared the proportions assigned to target color and target orientation, and the third ANOVA analyzed the proportions assigned to target shape and target orientation. In addition to this factor of stimulus dimension, saccade size and discriminability (LD vs. HD) were included as factors in the analysis.

All three ANOVAs yielded a significant discriminability $\left(\right.$ LD vs. HD) $\times$ stimulus dimension interaction (all $F_{\mathrm{S}} \geq$ 18.57, all $p s<.001$ ). This effect is illustrated in Figure 2, which shows the average proportions of fixations assigned to distractors sharing target color, shape, or orientation as a function of saccade size in both the LD (panel A) and HD (panel B) conditions. As an examination of this figure reveals, and the significant interactions confirm, the difference between any pair of dimensions varied with the discriminability of the shape dimension. Specifically, participants were significantly more likely to direct their eye movements to distractors sharing target color than to those sharing target shape or target orientation. In both cases, this difference was more pronounced in the LD condition (color vs. shape: all $t \mathrm{~s} \geq 12.49$, all $p \mathrm{~s}<.001$; colorvs. orientation: all $t \mathrm{~s} \geq 10.13$, all $p \mathrm{~s}<.001$ ) than in the HD condition (color vs. shape: all $t \mathrm{~s}>2.67$, all $p \mathrm{~s}<.05$; color vs. orientation: all $t \mathrm{~s} \geq 3.62$, all $p \mathrm{~s}<.01$ ). The proportions of eye movements assigned to distractors sharing target shape and target orientation did not differ significantly in the LD condition except for saccades greater than $6^{\circ}[t(31)=2.35, p<.05$; all other $t$ s $<1.7]$, while in the HD condition, the proportion assigned to target shape was consistently greater than the proportion assigned to target orientation (all $t \mathrm{~s} \geq 1.82$, all $p \mathrm{~s}<.08$ ). Thus, in the LD condition, it appears that participants relied heavily on color information to guide their eye movements during visual search, with shape and orientation information being little used. In the HD condition, par- 


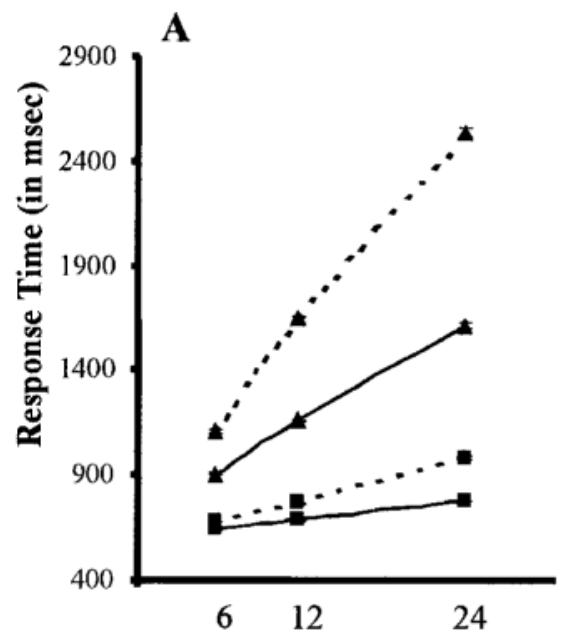

Display Size

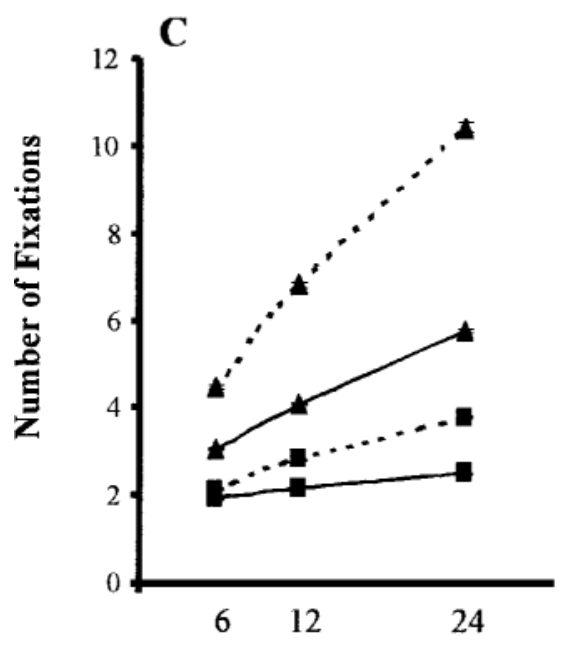

Display Size

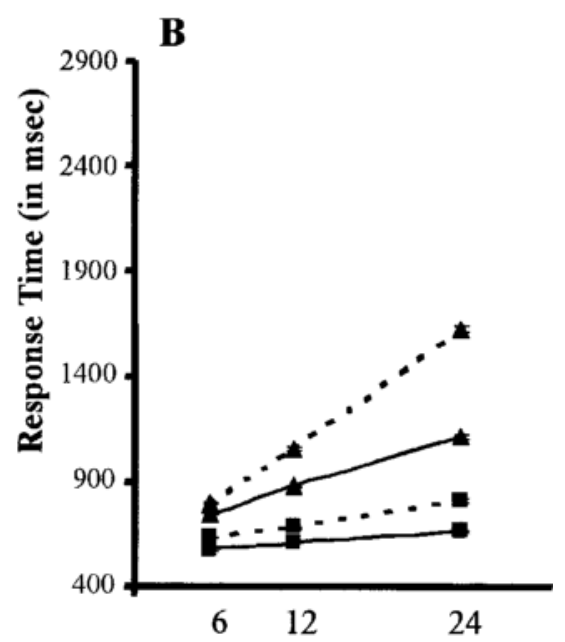

Display Size

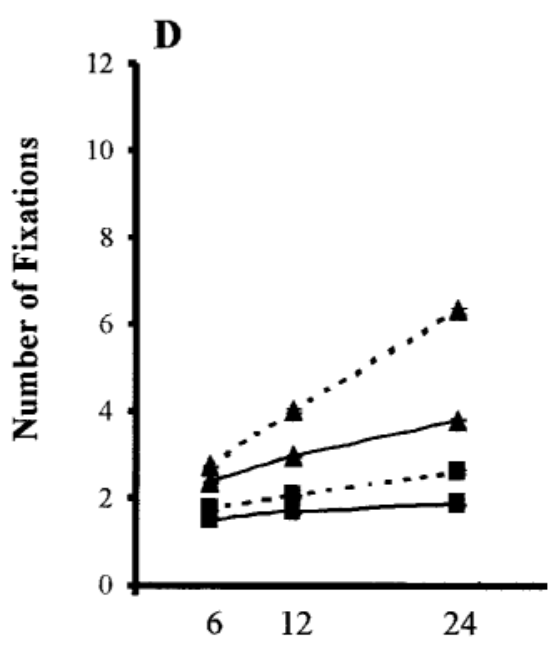

Display Size

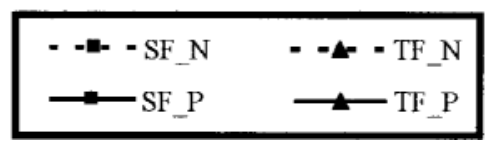

Figure 1. Average $( \pm S E M)$ response time and mean $( \pm S E M)$ number of fixations per trial as a function of display size on positive and negative trials in both the single-feature (SF) and twofeature (TF) search tasks in the low-discriminability condition and in the high-discriminability condition.

ticipants were still most likely to make use of color information; however, they now sometimes used shape information to guide their selection of saccadic endpoint. The main effect of saccade size was nonsignificant in all three analyses, as were all interactions with saccade size, indicating that patterns of guidance were not affected by this factor (all $F \mathrm{~s} \leq 1.55$ and all $p \mathrm{~s} \geq 0.16$ ).

Following Zelinsky (1996), the data were collapsed across saccade amplitude and a further analysis was conducted to see whether the observed pattern of selectivity varied with search progression by comparing the selectivity of first and second saccades (Table 1). For each stimulus dimension, a two-way ANOVA was performed, crossing discriminability (LD vs. HD) with search progression (i.e., first vs. second saccade). All three analyses yielded significant discriminability $\times$ search progression interactions [color: $F(1,62)=24.04, M S_{\mathrm{e}}=35.39$, $p<.001$; shape: $F(1,62)=4.56, M S_{\mathrm{e}}=26.99, p<.05$; orientation: $\left.F(1,62)=15.93, M S_{\mathrm{e}}=20.46, p<.001\right]$. Post hoc comparisons revealed that in the LD condition 


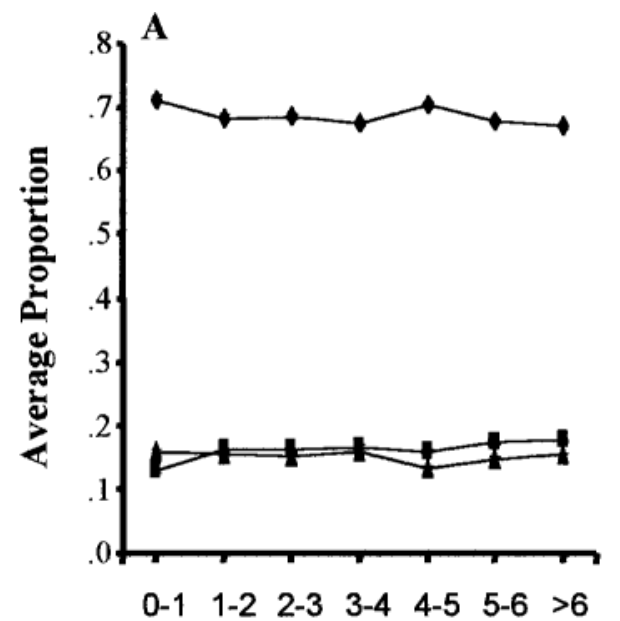

Saccade Size (in degrees)

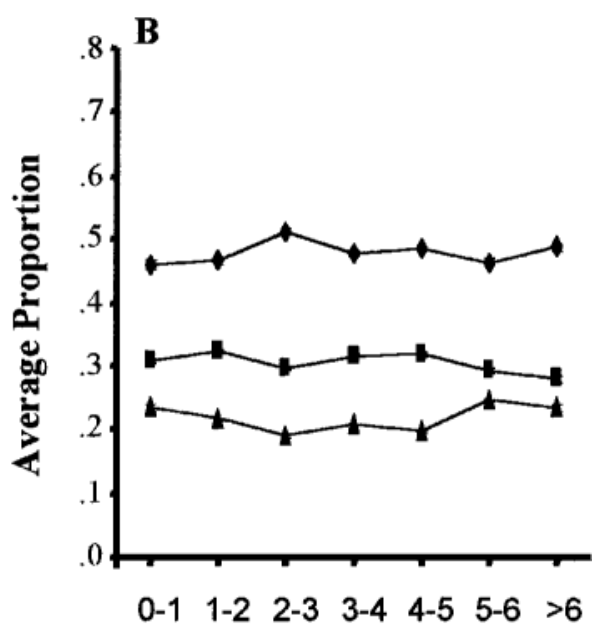

Saccade Size (in degrees)

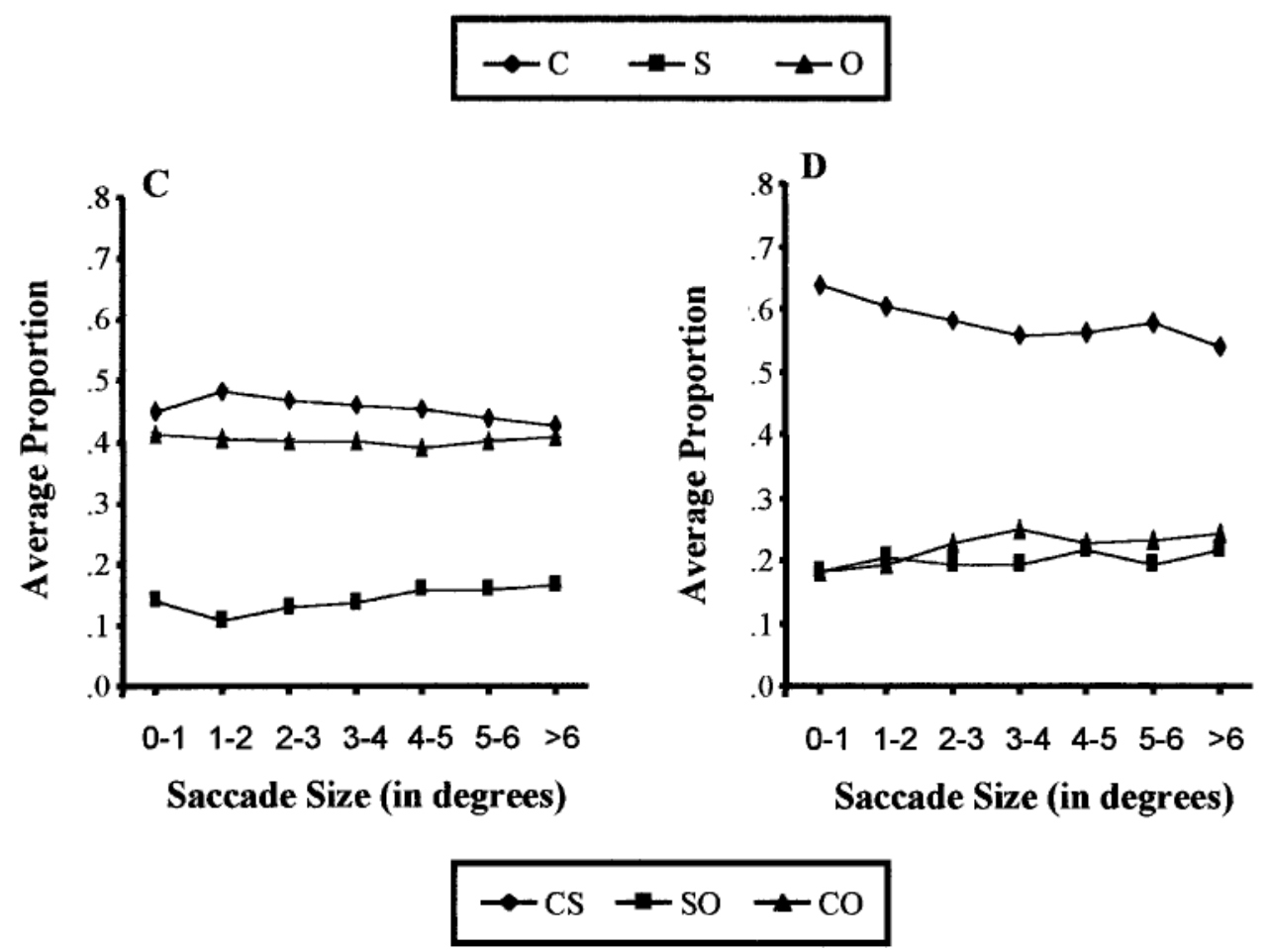

Figure 2. Average ( $\pm S E M)$ proportion of fixations assigned to distractors sharing target color $(\mathrm{C})$, shape $(S)$, or orientation (O) in the single-feature (SF) search task (Panel A: LD; Panel B: HD) and to distractors sharing target color shape (CS), color orientation (CO), or shape orientation (SO) in the two-feature search task Panel C: LD; Panel D: HD) as a function of saccade amplitude.

the proportion of saccades directed toward stimuli sharing target color increased significantly from the first to the second saccade, while the proportion of saccades directed toward shape and orientation showed a concomitant decrease. In the HD condition, the average proportion of saccades did not differ reliably between the first and second saccades; this was true for all three stimulus dimensions. This pattern of results suggests that when search is inefficient, as it was in the LD condition, the use of stimulus information to guide search may increase as search progresses.

To determine whether strength of selectivity of the first saccade varied with the duration of the latency to move, selectivity was compared for each participant for saccades following short (below the median) and long (above the median) latencies (Table 1). Across participants, short latencies to move lasted $177.18 \mathrm{msec}$, on average, whereas long latencies lasted an average of $300.33 \mathrm{msec}$. A sep- 
Table 1

Average Percent of Fixations and Standard Errors of the Mean by Condition and Task for First Saccades Following Short (Below Median) and Long (Above Median) Latency to Move, and for First, Second, and All Saccades

\begin{tabular}{|c|c|c|c|c|c|c|c|c|c|c|c|}
\hline \multirow[b]{2}{*}{ Condition } & \multirow[b]{2}{*}{ Task } & \multicolumn{2}{|c|}{ Short Latency } & \multicolumn{2}{|c|}{ Long Latency } & \multicolumn{2}{|c|}{ First Saccade } & \multicolumn{2}{|c|}{$\underline{\text { Second Saccade }}$} & \multicolumn{2}{|c|}{ All Saccades } \\
\hline & & $\%$ & SEM & $\%$ & SEM & $\%$ & SEM & $\%$ & $S E M$ & $\%$ & SEM \\
\hline \multirow[t]{6}{*}{ LD } & SF C & 65.95 & 1.89 & 62.08 & 1.89 & 64.21 & 0.25 & 72.47 & 0.24 & 67.34 & 0.20 \\
\hline & $\mathrm{S}$ & 16.66 & 1.05 & 20.36 & 0.98 & 18.35 & 0.14 & 14.85 & 0.16 & 16.88 & 0.11 \\
\hline & $\mathrm{O}$ & 17.38 & 1.26 & 17.55 & 1.42 & 17.43 & 0.18 & 12.67 & 0.13 & 15.78 & 0.11 \\
\hline & TF CS & 44.02 & 1.60 & 49.35 & 1.49 & 46.68 & 0.24 & 51.39 & 0.21 & 45.12 & 0.11 \\
\hline & $\mathrm{CO}$ & 37.53 & 1.42 & 34.39 & 1.47 & 35.96 & 0.21 & 36.32 & 0.20 & 40.54 & 0.10 \\
\hline & SO & 18.43 & 1.37 & 16.24 & 1.07 & 17.35 & 0.18 & 12.28 & 0.14 & 14.34 & 0.12 \\
\hline \multirow[t]{6}{*}{ HD } & $\mathrm{SF} \mathrm{C}$ & 52.04 & 1.84 & 47.00 & 2.11 & 49.50 & 0.30 & 47.45 & 0.43 & 47.52 & 0.33 \\
\hline & S & 28.52 & 1.53 & 30.93 & 1.78 & 29.75 & 0.26 & 30.17 & 0.34 & 30.46 & 0.25 \\
\hline & $\mathrm{O}$ & 19.42 & 1.15 & 22.05 & 1.26 & 20.74 & 0.18 & 22.36 & 0.25 & 22.02 & 0.16 \\
\hline & TF CS & 55.20 & 2.16 & 54.74 & 1.95 & 55.15 & 0.34 & 63.89 & 0.31 & 56.79 & 0.26 \\
\hline & $\mathrm{CO}$ & 25.04 & 1.23 & 24.80 & 1.38 & 24.74 & 0.21 & 19.42 & 0.19 & 23.21 & 0.14 \\
\hline & $\mathrm{SO}$ & 19.74 & 1.44 & 20.44 & 1.40 & 20.11 & 0.22 & 16.68 & 0.17 & 20.00 & 0.16 \\
\hline
\end{tabular}

Note-C, color; S, shape; O, orientation; CS, color shape; CO, color orientation; SO, shape orientation; LD, low discriminability; HD, high discriminability; SF, single-feature; TF, two-feature.

arate $2 \times 2$ ANOVA was performed for each stimulus dimension (color, shape, and orientation) that crossed discriminability (LD vs. HD) and latency (short vs. long). All three analyses yielded significant effects of discriminability [color: $F(1,62)=41.42, M S_{\mathrm{e}}=162.22, p<$ .001 ; shape: $F(1,62)=45.16, M S_{\mathrm{e}}=89.10, p<.001$; orientation: $\left.F(1,62)=5.22, M S_{\mathrm{e}}=65.92, p<.05\right]$. These effects are consistent with the results of the eccentricity analysis (above) and will not be discussed further. The main effect of latency was also significant for both color $\left[F(1,62)=8.11, M S_{\mathrm{e}}=78.31, p<.01\right]$ and shape $\left[F(1,62)=9.25, M S_{\mathrm{e}}=32.28, p<.005\right]$, but not for orientation $\left[F(1,62)=1.26, M S_{\mathrm{e}}=38.43, p=\right.$ n.s. $]$. Participants were more likely to fixate on distractors sharing target color following short initial latencies than following long initial latencies. An opposite pattern was observed for the shape dimension, with the proportion of saccades assigned to shape being greater following long as opposed to short initial latencies. This may suggest that color information from the periphery is available to guide search earlier as compared with shape information. It should be noted, however, that evidence of selectivity was observed following both short and long initial latencies; in both cases, participants directed saccades to stimuli sharing target color more often than predicted by chance (both $t \mathrm{~s} \geq 99.91$ and both $p \mathrm{~s}<.001$ ) while directing saccades toward stimuli sharing target shape and orientation less often than predicted by chance (all $t_{\mathrm{s}} \leq-50.89$ and all $\left.p_{\mathrm{s}}<.001\right)$.

TF search task. As was done for the SF search task, each saccade on negative trials was assigned to one of seven bins as a function of size. For each participant, the proportions of fixations assigned to distractors sharing target color and shape (CS), target color and orientation (CO), and target shape and orientation (SO) were then calculated for each bin. Average proportions are shown in Figure 2 (panel C: LD search condition; panel D: HD search condition). A series of three ANOVAs was then performed, crossing discriminability (LD vs. HD) with saccade amplitude (from $0^{\circ}-1^{\circ}$ to $>6^{\circ}$ ) and feature pair (i.e., $\mathrm{CS}$ vs. CO, CS vs. SO, and $\mathrm{CO}$ vs. $\mathrm{SO}$ ).

All three ANOVAs yielded three-way interactions that were at least marginally significant (all $p \mathrm{~s}<.075)$. These interactions disappeared, however, when information from the first two bins (i.e., $0^{\circ}-1^{\circ}$ and $1^{\circ}-2$ ) was dropped from the analyses (all $p$ s $>.27$ ). Since these bins contained the very short saccades, the effect may reflect difficulty in rejecting foveated distractors rather than preattentive guidance. Consequently, these interactions are of limited relevance in the present context.

Of greater importance are the discriminability $\times$ feature pair interactions, which were significant in all three analyses. An inspection of panels C and D in Figure 1 reveals that, in both the LD condition and the HD condition, CS distractors were fixated more often than SO distractors. This difference was slightly larger in the HD condition $\left[F(1,62)=5.81, M S_{\mathrm{e}}=.03, p<.05\right]$. The most dramatic shift between the two conditions has to do with the proportion of saccades assigned to distractors sharing target color and orientation. In the LD condition, the proportion of saccades assigned to $\mathrm{CO}$ was similar to the proportion assigned to CS, whereas, in the HD condition, proportion assigned to $\mathrm{CO}$ was similar to the proportion assigned to SO. As a result, the difference between $\mathrm{CS}$ and $\mathrm{CO}$ was much greater in the HD than in the $\mathrm{LD}$ condition $\left[F(1,62)=143.22, M S_{\mathrm{e}}=.02, p<.001\right]$, while the difference between $\mathrm{CO}$ and $\mathrm{SO}$ was much greater in the LD than in the HD condition $[F(1,62)=269.83$, $\left.M S_{\mathrm{e}}=.01, p<.001\right]$. All of these interactions clearly illustrate the greater utilization of shape information when the discriminability of that dimension is increased, as predicted earlier.

As in the SF task, the data from the TF task were collapsed across saccade amplitude, and patterns of selectivity for the first and second saccades were compared to see whether there was any change in guidance as search progressed (Table 1). A separate ANOVA was performed for each pair of stimulus dimensions, crossing discrim- 
inability (LD vs. HD) and search progression (i.e., first vs. second saccade). The analyses of both CS and CO revealed discriminability $\times$ saccade interactions [CS: $F(1,62)=2.86, M S_{\mathrm{e}}=45.47, p=.1 ; \mathrm{CO}: F(1,62)=7.82$, $\left.M S_{\mathrm{e}}=33.00, p<.01\right]$, while the analysis of SO yielded a significant main effect of saccade $[F(1,62)=27.71$, $\left.M S_{\mathrm{e}}=20.82, p<.001\right]$. In both the LD and the HD conditions, the proportion of saccades directed toward stimuli sharing target color and shape increased from the first to the second saccade, with this increase being greater in the HD condition. To offset this increase, the proportion of saccades directed to $\mathrm{CO}$ and $\mathrm{SO}$ showed concomitant decreases from the first to second saccade in the HD condition, whereas in the LD condition, a decrease was observed only in the proportion of saccades assigned to shape and orientation. These results are consistent with guidance becoming stronger as search progresses.

All latencies to move for each participant were divided into short (below median) and long (above median) latencies in a manner similar to that for the SF task. Once this was done, guidance of first saccades following short and long latencies could be compared (Table 1). On average, short latencies lasted for $165.37 \mathrm{msec}$, and long latencies lasted for $268.48 \mathrm{msec}$. A separate ANOVA was performed for each pair of stimulus dimensions (CS, CO, and SO), crossing discriminability (HD vs. LD) and latency (short vs. long). The discriminability $\times$ latency interaction was significant in the analysis of CS $[F(1,62)=$ $\left.7.06, M S_{\mathrm{e}}=38.04, p<.05\right]$. This reflects the fact that in the LD condition, the proportion of saccades assigned to CS was greater following long latencies as opposed to short latencies, whereas in the HD condition, there was no significant difference in the proportion of saccades assigned to CS following short and long initial latencies. Both of the main effects and the interaction were nonsignificant for the analysis of SO (all $F \mathrm{~s} \leq 2.93$ and all $p \mathrm{~s} \geq$ $.09)$. In the analysis of color and orientation, the main effect of discriminability was significant $[F(1,62)=44.07$, $\left.M S_{\mathrm{e}}=88.48, p<.001\right]$, reflecting a difference discussed earlier. This analysis reveals a small effect of the duration of latency to move on guidance, but it should be noted that selectivity was evident following both short and long initial latencies; in both cases, the proportion of saccades assigned to CS was significantly greater than chance (both $t \mathrm{~s} \geq 86.41$ and all $p \mathrm{~s}<.001$ ), whereas the proportions assigned to $\mathrm{CO}$ and $\mathrm{SO}$ were significantly smaller than chance (all $t \mathrm{~s} \leq-13.38$ and all $p \mathrm{~s}<.001$ ).

\section{Endpoint of First Saccade}

As noted, Zelinsky (1996) suggested that in addition to preattentive guidance, other cognitive strategies might influence the selection of saccadic endpoints. For example, Zelinsky found that on first saccades, participants tended to move their eyes toward stimuli in the upper lefthand quadrant of displays. To see whether such a tendency was present in the present data, displays were divided into quadrants and the proportion of first saccades directed to each quadrant was calculated for each participant. These proportions were then analyzed using ANOVAs that crossed discriminability (LD vs. HD) and quadrant (top left, top right, bottom left, bottom right); this was done separately for the SF and TF tasks.

When participants performed the SF search task in the LD condition, they were significantly more likely to direct first saccades toward the upper-left quadrant of stimulus displays (average proportion: 31.86 ) than to any other quadrant (average proportions ranging from 20.82 to 24.71), whereas in the HD condition, no such preference was evident (all average proportions between 21.92 and $26.29)\left[F(3,186)=3.99, M S_{\mathrm{e}}=105.06, p<.01\right]$. In contrast, in the TF search task, participants showed a reliable tendency to direct initial saccades toward the upper-left quadrant in both the LD and HD conditions, with this effect being stronger in the LD search condition (upper left: 41.96; remaining quadrants: $17.54-21.67$ ) than in the HD search condition (upper left: 32.30 ; remaining quadrants: 21.69-23.12) $\left[F(3,186)=3.80, M S_{\mathrm{e}}=180.14, p<.05\right]$.

It appears that when search is efficient (e.g., SF task in the HD condition), participants are able to effectively use preattentive information to guide the search process, and as a result, do not need to resort to other strategies. In contrast, when search is inefficient (e.g., TF task in the LD condition), it appears that preattentive information is less informative, leading participants to draw upon alternative strategies. When the efficiency of a search process falls between these two extremes (e.g., SF task in the LD condition and TF task in the HD condition), it appears that participants use a combination of search strategies.

\section{DISCUSSION}

The results from the analysis of RT and number of fixations per trial suggest that search was more efficient in the SF task than in the TF task. This finding is consistent with previous work using triple conjunctions (Quinlan \& Humphreys, 1987; Treisman \& Sato, 1990; Wolfe et al., 1989) and provides additional evidence that targetdistractor similarity is an important determinant of search efficiency, as proposed by many theories of visual search (e.g., Cave \& Wolfe, 1990; Duncan \& Humphreys, 1989, 1992; Treisman, 1988; Treisman \& Sato, 1990; Wolfe, 1994). In addition, search was more efficient in the HD condition than in the LD condition, suggesting that search efficiency varies with the discriminability of the feature values utilized within a stimulus dimension (Wolfe, 1994, 1998).

The evidence of selectivity in the guidance of eye movements during visual search in the present study is consistent with the results of other eye movement studies (e.g., Findlay, 1997; Luria \& Strauss, 1975; Motter \& Belky, 1998; Scialfa \& Joffe, 1998; Shen, Reingold, \& Pomplun, 2000; L. G. Williams, 1967), as well as RT studies (e.g., Egeth, Virzi, \& Garbart, 1984; Kaptein, Theeuwes, \& van der Heijden, 1995; Poisson \& Wilkinson, 1992; Smith, 1962; Zohary \& Hochstein, 1989), as is the finding that participants relied heavily on information con- 
cerning target color (e.g., RT studies: Egeth et al., 1984; Kaptein et al., 1995; Poisson \& Wilkinson, 1992; Smith, 1962; Zohary \& Hochstein, 1989; e.g., eye movement studies: Luria \& Strauss, 1975; Motter \& Belky, 1998; L. G. Williams, 1967). The present results extend previous eye movement research on this topic in a number of ways. Most importantly, this study demonstrates that guidance of eye movements can be observed across a wide range of saccadic amplitudes. This finding is inconsistent with Findlay and Gilchrist's (1998) suggestion that the weak guidance observed in Zelinsky's (1996) study was due to the influence of stimulus proximity overriding the presence or absence of features shared with the target. In addition, the finding that guidance is observed across a wide range of saccadic amplitudes rules out the possibility that evidence of selectivity or guidance results from a tendency for participants to refixate distractors that are similar to the target.

The present study also extends earlier work by showing that although color may play an important role in guidance of visual search, more generally, the discriminability of the feature values on a particular stimulus dimension may determine the extent to which information from that dimension contributes to the guidance of visual search. For example, in the SF task, when the stimuli were changed from Es and Fs (LD condition) to Cs and Ts (HD condition), the proportion of saccades directed at distractors sharing target shape went from 0.17 to 0.30 . In the TF task, the difference between the proportion of saccades directed at distractors sharing target color and shape and the proportion sharing target color and orientation increased from 0.05 (LD condition) to 0.34 (HD condition). One implication of these findings is that any study looking at the relative contribution of different stimulus dimensions to guidance must be preceded by a psychophysical experiment to match stimulus discriminability across dimensions.

An examination of patterns of guidance in both the SF and TF triple conjunction search tasks made it possible to demonstrate simultaneous guidance on more than one stimulus dimension. Performance in the SF task allowed an estimation of the extent to which information from each single dimension was used to guide search. The analysis of the TF data revealed that performance in this task cannot be explained as a simple combination of the estimates from the SF task. For example, in the SF task in the HD condition, the average proportion of saccades assigned to color (0.48) was greater than that assigned to shape (0.30), which in turn was greater than that assigned to orientation (0.22). In the corresponding TF task, in the absence of simultaneous guidance, the average proportion of saccades directed at $\mathrm{CO}$ should be greater than the proportion directed at SO. Instead, the average proportions of saccades assigned to $\mathrm{CO}$ and $\mathrm{SO}$ were roughly equal (CO: 0.23; SO: 0.20).

Another purpose of the present study was to evaluate the finding by Hooge and Erkelens (1999) that the strength of saccadic guidance depends on the duration of the pre- ceding fixation. To this end, for each participant a median split of all latencies to move was performed, following which the guidance of the first saccade was examined for both short (below median) and long (above median) latencies. The results of this analysis were mixed; in the SF task, guidance was slightly stronger following short rather than long latencies, whereas in the TF task, the opposite was the case. More importantly, the observed differences were small, and guidance of saccades following both short and long latencies to move was robust for both tasks. Given that the procedure used in the present experiment differed substantially from that employed by Hooge and Erkelens, further research would be required to fully explain these contrasting results.

The present study replicated Zelinsky's (1996) finding that participants may rely on strategies other than the use of preattentive information in an attempt to maximize search efficiency. Zelinsky found that participants tended to direct initial saccades toward stimuli in the upper left-hand quadrant of the stimulus array. The results of the present study extended this finding by demonstrating that reliance on this strategy was mediated by search efficiency. Search efficiency, as indicated by RT and number of fixations per trial, was lowest for the TF task in the LD search condition, and it was in this condition that participants were more likely to direct first saccades toward the upper left-hand quadrant of a display ( 0.42 of first saccades). In contrast, the RT and numberof-fixation data show that search was most efficient for the SF task in the HD condition, and participants showed no significant preference for directing their first eye movements to the upper left-hand quadrant ( 0.26 of first saccades). Finally, search efficiency for the SF task in the LD condition and for the TF task in the HD condition fell between these two extremes, as did the probability that participants would direct their first eye movements toward the upper left-hand quadrant (0.32 in both cases). Taken together, these findings suggest that the likelihood of participants making use of alternative search strategies increases as search efficiency decreases. Further research will be needed to specify more precisely the relation between search efficiency and the use of strategies.

The guidance observed in the present study was very robust, being observed across participants, across display sizes, and across a wide range of saccadic eccentricities. Guidance was also observed when first and second saccades were considered separately, with the strength of selectivity increasing throughout the course of a trial. These findings are consistent with prior demonstrations of saccadic selectivity (Findlay, 1997; Luria \& Strauss, 1975; Motter \& Belky, 1998; Scialfa \& Joffe, 1998; L. G. Williams, 1967) and contrast with the weak selectivity observed by Zelinsky (1996). Several factors may account for this discrepancy across studies. To begin with, it should be noted that although the differences observed in Zelinsky's experiment were small and sometimes nonsignificant, saccades were still more likely to be directed at "similar" distractors (approximately 0.55 of saccades) 
than at "dissimilar" distractors (approximately 0.45 of saccades) on negative trials. Thus, Zelinsky's findings do provide weak evidence for selectivity, as he acknowledged. In addition, Zelinsky compared "similar" and "dissimilar" distractors, with "similar" distractors being of two types (same color vs. same orientation). When calculating the percentage of saccades directed at "similar" distractors, Zelinsky did not distinguish between saccades directed at distractors sharing target color and those sharing target orientation. In the present study, participants were found to mainly rely on information concerning target color to guide search, but to make little use of information concerning orientation (see also Motter \& Belky, 1998). Assuming that participants in Zelinsky's study behaved in a similar manner, lumping these two types of distractors together during analysis may have led to an underestimation of the strength of guidance in his study.

The present results are consistent with the guided search model (e.g., Cave \& Wolfe, 1990; Wolfe, 1994, 1996; Wolfe et al., 1989) and, like other findings (e.g., McLeod et al., 1988; Nagy \& Sanchez, 1990; Nakayama \& Silverman, 1986; Steinman, 1987; Theeuwes \& Kooi, 1994; Wolfe et al., 1992), are inconsistent with early versions of the feature integration theory (e.g., Treisman \& Gelade, 1980; Treisman et al., 1977). A comprehensive discussion of the implications of the present results for recent theoretical frameworks (e.g., Bundesen, 1990; Duncan \& Humphreys, 1989, 1992; Humphreys \& Mueller, 1993; Mueller \& Humphreys, 1993; Mueller, Humphreys, \& Donnelly, 1994; Palmer, 1994, 1995) and current issues concerning visual search is beyond the scope of this paper. Nevertheless, in order to illustrate the potential applications of our results, we will consider two specific examples. Recently, there has been a great deal of interest in the effect of stimulus familiarity on visual search (e.g., Kuehn \& Jolicœur, 1994; Polke \& Farah, 1995; Shen \& Reingold, 1999; Wang, Cavanagh, \& Green, 1994). Determining whether familiarity can preattentively guide visual search, as did the dimensions of color and shape in the present experiment, could have important implications for future research. In the present study, the stimuli were letters that were presented either upright (i.e., familiar) or rotated $90^{\circ}$ clockwise (i.e., unfamiliar). Thus, our manipulation of orientation affected stimulus familiarity. The finding that orientation had little impact on selectivity of eye movements therefore argues against a strong effect of familiarity on the preattentive guidance in visual search. This is consistent with the results from a more direct test of this issue carried out by Shen and Reingold (1999). They examined the proportion of fixations directed at familiar and unfamiliar distractors in a visual search task. They found evidence of selectivity in patterns of eye movements only when the target stimulus was unfamiliar. In this case, participants showed a small but significant bias to fixate unfamiliar as opposed to familiar distractors. The present results, taken together with the work by Shen and Reingold, suggest that unlike other dimensions such as color and shape, stimulus familiarity may have little effect on guidance of visual search.

As a further illustration of potential applications of the present findings, consider the sequential attentional model proposed by Henderson (1992). According to this model, at the start of a fixation, attention is focused on the currently fixated stimulus. Once processing of this stimulus reaches a particular stage or threshold, the attentional focus is shifted to another stimulus in the visual array. This shift of attention signals the eye movement system to program and execute a saccade to the newly attended stimulus location. Interestingly, the sequential attentional model proposes an account of attentional control similar to that envisioned in the guided search theory. The sequential attentional model proposes that shifts of attention are determined by a map of stimulus locations, which receives input from preattentive processes. Locations within this map are thought to be differentially weighted so that, when shifted, the attentional focus is directed to the most heavily weighted location. Thus, when considered together, the two models provide one possible explanation of how the attention and eye movement systems might interact in visual search, with the guided search theory focusing on attentional processes, while the sequential attentional model demonstrates how such attentional processes might be realized as patterns of eye movements.

In conclusion, the present study joins a growing body of literature in which studying patterns of eye movements has greatly contributed to our knowledge of both visual search (e.g., Findlay, 1997; Gould, 1967; Gould \& Schaffer, 1967; Jacobs, 1987; Luria \& Strauss, 1975; Nattkemper \& Prinz, 1984; Rayner \& Fisher, 1987; Scialfa \& Joffe, 1998; Shen et al., 2000; D. E. Williams, Reingold, Moscovitch, \& Behrmann, 1997; L. G. Williams, 1967; Zelinksy, 1996; Zelinsky \& Sheinberg, 1995, 1997; for review, see Rayner, 1998) and visual attention (e.g., Henderson, 1993; Hodgson \& Mueller, 1995; Hoffman \& Subramaniam, 1995; Kowler, Anderson, Dosher, \& Blaser, 1995; Schneider \& Deubel, 1995; Shepherd, Findlay, \& Hockey, 1986; for review, see Hoffman, 1998).

\section{REFERENCES}

Bundesen, C. (1990). A theory of visual attention. Psychological Review, 97, 523-547.

CAVE, K. R., \& Wolfe, J. M. (1990). Modeling the role of parallel processing in visual search. Cognitive Psychology, 22, 225-271.

Duncan, J., \& Humphreys, G.W. (1989). Visual search and stimulus similarity. Psychological Review, 96, 433-458.

Duncan, J., \& Humphreys, G.W. (1992). Beyond the search surface: Visual search and attentional engagement. Journal of Experimental Psychology: Human Perception \& Performance, 18, 578-588.

Egeth, H. E., Virzi, R. A., \& Garbart, H. (1984). Searching for conjunctively defined targets. Journal of Experimental Psychology: Human Perception \& Performance, 10, 32-39.

FindLaY, J. M. (1980). The visual stimulus for saccadic eye movements in human observers. Perception, 9, 7-20.

FindLAY, J. M. (1997). Saccade target selection during visual search. Vision Research, 37, 617-631. 
Findlay, J. M., \& Gilchrist, J. D. (1998). Eye guidance and visual search. In G. Underwood (Ed.), Eye guidance in reading and scene perception (pp. 295-312). Amsterdam: Elsevier.

Gould, J. D. (1967). Pattern recognition and eye-movement parameters. Perception \& Psychophysics, 2, 399-407.

Gould, J. D., \& Schaffer, A. (1967). Eye-movement parameters in pattern recognition. Journal of Experimental Psychology, 74, 225-229.

Henderson, J. M. (1992). Visual attention and eye-movement control during reading and picture viewing. In K. Rayner(Ed.), Eye movements and visual cognition: Scene perception and reading (pp. 260-283). New York: Springer-Verlag.

Henderson, J. M. (1993). Visual attention and saccadic eye movements. In G. d'Ydewalle \& J. van Rensbergen (Eds.), Perception and cognition: Advances in eye movement research (pp.37-50). Amsterdam: Elsevier.

Hodgson, T. L., \& Mueller, H. J. (1995). Evidence relating to premotor theories of visuospatial attention. In J. M. Findlay, R. Walker, $\&$ R. W. Kentridge (Eds.), Eye movement research: Mechanisms, processes and applications (pp. 305-316). Amsterdam: Elsevier.

Hoffman, J. E. (1998). Visual attention and eye movements. In H. Pashler (Ed.), Attention (pp. 119-153). Hove, U.K.: Erlbaum.

Hoffman, J. E., \& Subramaniam, B. (1995). The role of visual attention in saccadic eye movements. Perception \& Psychophysics, 57, 787-795.

Hooge, I. T., \& ERKelens, C. J. (1999). Peripheral vision and oculomotor control during visual search. Vision Research, 39, 1567-1575.

Humphreys, G. W., \& Mueller, H. J. (1993). Search via recursive rejection (SERR): A connectionist model of visual search. Cognitive Psychology, 25, 43-110.

JACOBS, A. M. (1987). Toward a model of eye movement control in visual search. In J. K. O'Regan \& A. Lévy-Schoen (Eds.), Eye movements: From physiology to cognition (pp. 275-284). Amsterdam: Elsevier.

Kaptein, N. A., Theeuwes, J., \& VAn der Heijden, A. H. C. (1995). Search for a conjunctively defined target can be selectively limited to a color-defined subset of elements. Journal of Experimental Psychology: Human Perception \& Performance, 21, 1053-1069.

Kowler, E., Anderson, E., Dosher, B., \& Blaser, E. (1995). The role of attention in the programming of saccades. Vision Research, 35, 1897-1916.

Kuehn, S. M., \& Jolicceur, P. (1994). Impact of quality of the image, orientation, and similarity of the stimuli on visual search for faces. Perception, 23, 95-122.

Luria, S. M., \& STRAuSS, M. S. (1975). Eye movements during search for coded and uncoded targets. Perception \& Psychophysics, 17, 303-308.

McLeod, P., Driver, J., \& Crisp, J. (1988). Visual search for a conjunction of movement and form is parallel. Nature, 332, 154-155.

Motter, B. C., \& BeLKy, E. J. (1998). The guidance of eye movements during active visual search. Vision Research, 38, 1805-1815.

Mueller, H. J., \& Humphreys, G. W. (1993). A connectionist model of visual search for simple form conjunctions. In D. Brogan, A. Gale, \& K. Carr (Eds.), Visual search 2 (pp. 61-71). London: Taylor \& Francis.

Mueller, H. J., Humphreys, G. W., \& Donnelly, N. (1994). Search via recursive rejection (SERR): Visual search for single and dual formconjunction targets. Journal of Experimental Psychology: Human Perception \& Performance, 20, 235-258.

Nagy, A. L., \& Sanchez, R. R. (1990). Critical color differences determined with a visual search task. Journal of the Optical Society of America A, 7, 1209-1217.

Nakayama, K., \& Silverman, G. H. (1986). Serial and parallel processing of visual feature conjunctions. Nature, 320, 264-265.

Natt Kemper, D., \& Prinz, D. (1984). Costs and benefits of redundancy in visual search. In A. G. Gale \& F. W. Johnson (Eds.), Theoretical and applied aspects of eye movement research (pp. 343-351). Amsterdam: Elsevier.

Palmer, J. (1994). Set-size effects in visual search: The effect of attention is independent of the stimulus for simple tasks. Vision Research, 34, 1703-1721.

Palmer, J. (1995). Attention in visual search: Distinguishing four causes of a set-size effect. Current Directions in Psychological Science, 4, 118-123.

Poisson, M. E., \& WiLkinson, F. (1992). Distractor ratio and grouping processes in visual conjunction search. Perception, 21, 21-38.

Polke, T. A., \& FARAH, M. J. (1995). Late experience alters vision. $\mathrm{Na}$ ture, 376, 648-649.
Quinlan, P. T., \& Humphreys, G. W. (1987). Visual search for targets defined by combinations of color, shape, and size: An examination of the task constraints on feature and conjunction searches. Perception \& Psychophysics, 41, 455-472.

RAYNER, K. (1998). Eye movements in reading and information processing: Twenty years of research. Psychological Bulletin, 124, 372-422.

RAYNER, K., \& Fisher, D. L. (1987). Eye movements and the perceptual span during visual search. In J. K. O'Regan \& A. Lévy-Schoen (Eds.), Eye movements: From physiology to cognition (pp. 293-302). Amsterdam: Elsevier.

Schneider, W. X., \& Deubel, H. (1995). Visual attention and saccadic eye movements: Evidence for obligatory and selective spatial coupling. In J. M. Findlay, R. Walker, \& R. W. Kentridge (Eds.), Eye movement research: Mechanisms, processes and applications (pp. 317-324). Amsterdam: Elsevier.

Scialfa, C. T., \& Joffe, K. M. (1998). Response times and eye movements in feature and conjunction search as a function of target eccentricity. Perception \& Psychophysics, 60, 1067-1082.

Shen, J., \& ReIngold, E. M. (1999). Saccadic selectivity during visual search: The effects of shape and stimulus familiarity. In M. Hahn \& S. C. Stoness (Eds.), Proceedings of the Twenty-First Annual Conference of the Cognitive Science Society (pp. 649-652). Mahwah, NJ: Erlbaum.

Shen, J., Reingold, E. M., \& Pomplun, M. (2000). Distractor ratio influences patterns of eye movements during visual search. Perception, 29, 241-250.

Shepherd, M., Findlay, J. M., \& Hockey, R. J. (1986). The relationship between eye movements and spatial attention. Quarterly Journal of Experimental Psychology, 38A, 475-491.

S Mith, S. L. (1962). Color coding and visual search. Journal of Experimental Psychology, 64, 434-440.

STAMPE, D. (1993). Heuristic filtering and reliable calibration methods for video-based pupil tracking systems. Behavior Research Methods, Instruments, \& Computers, 25, 137-142.

Steinman, S. B. (1987). Serial and parallel search in pattern vision. Perception, 16, 389-398.

Theeuwes, J., \& KooI, F. L. (1994). Parallel search for a conjunction of contrast polarity and shape. Vision Research, 34, 3013-3016.

Treisman, A. (1988). Features and objects: The Fourteenth Bartlett Memorial Lecture. Quarterly Journal of Experimental Psychology, 40A, 201-237.

Treisman, A. (1991). Search, similarity, and integration of features between and within dimensions. Journal of Experimental Psychology: Human Perception \& Performance, 17, 652-676.

Treisman, A. (1993a). The perception of features and objects. In A. Baddeley \& L. Weiskrantz (Eds.), Attention: Selection, awareness, and control: A tribute to Donald Broadbent (pp. 5-35). Oxford: Oxford University Press, Clarendon Press.

Treisman, A. (1993b). Representing visual objects. In D. E. Meyer \& S. Kornblum (Eds.), Attention and performance XIV: Synergies in experimental psychology, artificial intelligence, and cognitive neuroscience (pp. 163-175). Cambridge, MA: MIT Press.

Treisman, A., \& Gelade, G. (1980). A feature integration theory of attention. Cognitive Psychology, 12, 97-136.

Treisman, A., \& Gormican, S. (1988). Feature analysis in early vision: Evidence from search asymmetries. Psychological Review, 95, 15-48.

Treisman, A., \& Sato, S. (1990). Conjunction search revisited. Journal of Experimental Psychology: Human Perception \& Performance, 16, 459-478.

Treisman, A., Sykes, M., \& Gelade, G. (1977). Selective attention and stimulus integration. In S. Dornic (Ed.), Attention and performance VI (pp. 280-292). Hillsdale, NJ: Erlbaum.

WANG, Q., Cavanagh, P., \& GReEn, M. (1994). Familiarity and pop-out in visual search. Perception \& Psychophysics, 56, 495-500.

Williams, D. E., Reingold, E. M., Moscovitch, M., \& Behrmann, M. (1997). Patterns of eye movements during parallel and serial visual search tasks. Canadian Journal of Experimental Psychology, 51, 151-164.

Williams, L. G. (1967). The effects of target specification on objects fixated during visual search. Acta Psychologica, 27, 355-360.

Wolfe, J. M. (1994). Guided Search 2.0: A revised model of visual search. Psychonomic Bulletin \& Review, 1, 202-238. 
Wolfe, J. M. (1996). Extending guided search: Why guided search needs a preattentive "item map.” In A. F. Kramer, M. G. H. Coles, \& G. D. Logan (Eds.), Converging operations in the study of visual attention (pp. 247-270). Washington, DC: American Psychological Association.

Wolfe, J. M. (1998). Visual search. In H. Pashler (Ed.), Attention (pp. 1373). Hove, U.K.: Erlbaum.

Wolfe, J. M., Cave, K. R., \& Franzel, S. L. (1989). Guided search: An alternative to the feature integration model for visual search. Journal of Experimental Psychology: Human Perception \& Performance, 15, 419-433.

Wolfe, J. M., Friedman-Hill, S. R., Stewart, M. I., \& O'Connell, K. M. (1992). The role of categorization in visual search for orientation. Journal of Experimental Psychology: Human Perception \& Performance, 18, 34-49.

Zelinsky, G. J. (1996). Using eye saccades to assess the selectivity of search movements. Vision Research, 36, 2177-2187.

Zelinsky, G. J., \& Sheinberg, D. (1995). Why some search tasks take longer than others: Using eye movements to redef ine reaction times. In J. M. Findlay, R. Walker, \& R. W. Kentridge (Eds.), Eye movement research: Mechanisms, processes and applications (pp. 325-336). Amsterdam: Elsevier.

Zelinsky, G. J., \& Sheinberg, D. L. (1997). Eye movements during parallel-serial visual search. Journal of Experimental Psychology: Human Perception \& Performance, 23, 244-262.

Zohary, E., \& HochsteIn, S. (1989). How serial is serial processing in vision? Perception, 18, 191-200.

\section{NOTE}

1. This was true for all analyses except for the saccadic eccentricity analysis in the SF search task. Three participants had to be dropped from this analysis due to empty data cells. This resulted in unequal sample sizes, meaning that the Newman-Keuls test could not be used. As a result, for this analysis, significant effects were further explored using repeated measures $t$ tests.

(Manuscript received May 7, 2000; revision accepted for publication May 23, 2000.) 